\title{
Patellar luxation in dogs
}

This article was published in the following Dove Press journal:

Veterinary Medicine: Research and Reports

\section{Francesco Di Dona \\ Giovanni Della Valle \\ Gerardo Fatone}

Department of Veterinary Medicine and Animal Productions, University of Napoli “Federico II", Naples, Italy
Correspondence: Francesco Di Dona Department of Veterinary Medicine and Animal Productions, University of Napoli "Federico II", Via F. Delpino I, 80I38 Naples, Italy

Tel/fax +39 8I 253 604I

Email francesco.didona@unina.it
Abstract: Patellar luxation is a common orthopedic problem in dogs. Medial luxation is more common than lateral luxation and is usually diagnosed in dogs of small breed. Diagnosis is based on clinical evidence of patellar instability; however, diagnostic imaging is required to assess the amount of skeletal deformity and then the most appropriate method of treatment. Surgical options include both soft tissue and osseous techniques, however, in most of the cases, a combination of more procedures is used to achieve the correction of the luxation. Complication rate is generally low and the most common complications include reluxation and implant-associated complications. Prognosis is generally favorable, with most of the dogs returning to normal limb function. This article describes patellar luxation features in dogs, including clinical presentation, diagnosis, and treatment options available.

Keywords: medial patellar luxation, lateral patellar luxation, stifle, pelvic limb, dog, small breeds, canine

\section{Introduction}

Patellar luxation is one of the most common causes of lameness in the dog. Both large and small breed dogs may be affected; the disease may be seen in cats as well. ${ }^{1-4}$ The luxation can be medial, lateral, or bidirectional. The vast majority of luxations are medial and diagnosed in small breed dogs. Lateral luxation occurs less frequently and is commonly diagnosed in large or giant breed dogs..$^{5-11}$ The reported incidence of medial patellar luxation in small breed dogs is 12 times higher compared with large breed dogs. ${ }^{12}$

Most of the prevalence studies have identified that females are more predisposed to develop this disease than males. ${ }^{6,7,12-14}$ Neuter status seems to be a risk factor, with neutered dogs having about 3 times the odds of developing patellar luxation. ${ }^{14,15}$ Most of the dogs are affected unilaterally; however, bilateral luxation may be a common finding. ${ }^{7,16}$ Patellar luxation is a typical disease of young dogs, but clinical signs become often evident as the animal grows. For this reason, most luxations are diagnosed in young adults, generally within 3 years of age. ${ }^{13,14,17}$

Most common breeds affected by patellar luxation include: Poodle, Pomeranian, Yorkshire Terrier, Chihuahua, French Bulldog, Lhasa Apso, Cavalier King Charles Spaniel, Bichon, Pug, Bulldog, West Highland White Terrier, Jack Russell Terrier, and Shit-tzu. ${ }^{13,14,16,17}$

Genomic studies have identified chromosomes anomalies implicated in patellar luxation in specific dog breeds, such as the Dutch Flat-coated Retriever and Pomeranian. ${ }^{18,19}$ 
Patellar luxation in dogs has been graded as follows (Figure 1). ${ }^{5}$

- Grade I - patella can be manually luxated but returns to normal position when released.

- Grade II - patella luxates with stifle flexion or on manual manipulation and remains luxated until stifle extension or manual replacement occurs.

- Grade III - patella luxated continually, and can be manually replaced but will reluxate spontaneously when manual pressure is removed.

- Grade IV - patella luxated continually and cannot be manually replaced.

\section{Etiology and pathogenesis}

Patellar luxation is a congenital/developmental disorder, but it could be secondary to traumatic accident causing tearing or stretching of the joint capsule and fascia, leading to femoropatellar instability. Occasionally, patellar luxation may occur as a complication secondary to treatment of cranial cruciate ligament (CrCL) disease or fractures involving femur or tibia. ${ }^{16}$

Although the underlying cause of patellar luxation is still not completely understood, malalignment of the quadriceps mechanism seems to have a key role in the development of this disease. The quadriceps muscle group, patella, trochlear groove, patellar ligament, and tibial tuberosity are all part of the extensor mechanism of the stifle. Any abnormality of this mechanism can lead, during the growth period, to anatomical changes of distal femur and proximal tibia and subsequent patellar instability. However, preexisting patellar luxation may affect the normal quadriceps mechanism, thus being the cause of the secondary bone changes. The absence of the physiological pressure that the patella exerts on the articular cartilage of the trochlear groove during growth, may prevent the development of an adequately deep and wide groove (trochlear hypoplasia). ${ }^{20,21}$

Decreased angle of inclination of the femoral neck, may cause laxity of the extensor mechanism, as well hip luxation or femoral head excision. Altered anteversion angle has also been postulated as a condition that leads to stifle joint abnormalities. $^{22}$

Deformities of the stifle include the following: 1) distal femoral varus or valgus; 2) external or internal torsion of the distal femur; 3) proximal tibial varus or valgus; 4) internal or external tibial torsion; and 5) shallow trochlear sulcus. $^{21-25}$

\section{Clinical presentation}

Clinical signs of dogs with patellar luxation can vary from animal to animal and are only partially related to the degree of concomitant skeletal deformities. Lameness may be intermittent or continuous, and usually is a mild-to-moderate

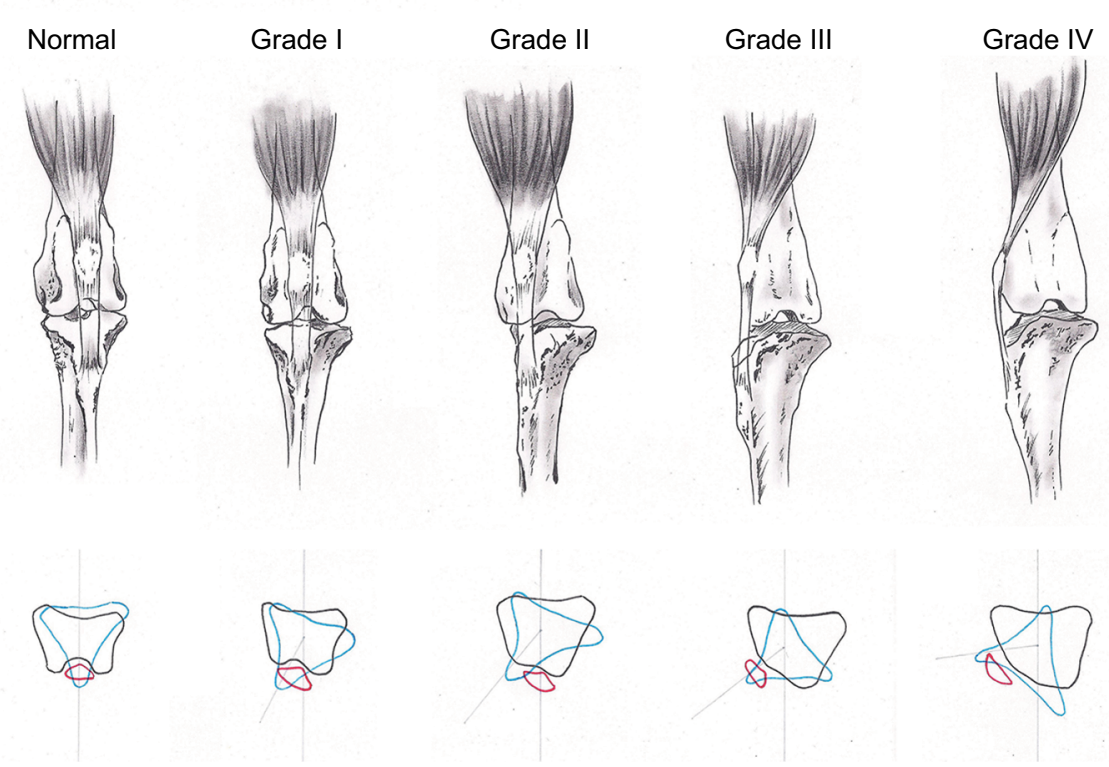

Figure I Skeletal abnormalities associated with patellar luxation.

Notes: On the left, a normal hindlimb cranial view; note the quadriceps mechanism centered over the femur as well the patella seated in the trochlear groove. Grade I-IV images demonstrate how the abnormal relation between the distal femur and the proximal tibia affect the position of the patella. Transverse views show the internal rotation of the tibia with regard to the femur and the subsequent luxation of the patella that in grades III and IV override completely the medial trochlear ridge. Also grade III and IV luxations are characterized by a shallow groove. 
weightbearing lameness with occasional lifting of the limb. A common finding during gait assessment is the attempt of the dog to stretch the leg backward to allow the patella to seat back in the groove, when luxated. Lateral dislocation produces a more serious symptomatology than the medial one. Whereas traumatic luxation has sudden onset and is, usually, associated with a traumatic accident, such as jump, fall from height, road traffic accident, etc..$^{20,22,26}$

Dogs with grade I patellar luxation are generally asymptomatic; however, they can present occasionally a "skipping" type lameness typical of dogs with grade II patellar luxation. Sometimes, on examination, a mild internal rotation of the tibia and abduction of the hock can be observed. Grade II luxation can evolve to a grade III due to the progressive erosion of the trochlear ridge and lead to more severe clinical signs, as a result of chronic degenerative joint changes. ${ }^{22}$

Grade III and IV patellar luxations are usually characterized by persistent lameness and abnormal posture. Bilateral medial luxation can determine abnormal, "crouched" gait rather than lameness, leg constantly semiflexed and internally rotated, as well varus deformity of the stifle. In case of lateral dislocation, the stifles appear to be close to each other while the distal extremities are abducted (valgus). ${ }^{20,27}$

Some dogs with subclinical patellar luxation can develop acute severe lameness due to concomitant $\mathrm{CrCL}$ injury as result of the degenerative joint changes and joint instability. Concurrent rupture of the $\mathrm{CrCL}$ has been reported in a study in $41 \%$ of the stifle joints of dogs with medial patellar luxation. Risk factors included age, grade of luxation, and advanced degenerative joint disease (DJD). ${ }^{28}$

\section{Diagnosis}

Careful physical examination is necessary to establish the grade of luxation and rule out concomitant affection that could cause hindlimb lameness. Orthopedic examination consists of gait evaluation, joint flexion-extension movements and range of motion evaluation, and tendency of the patella to luxation and grading. At walk and trot, it is possible to determine the degree of lameness as well to identify obvious skeletal deformities. Physical examination is performed at the beginning with the patient standing. This is optimal to evaluate the symmetry between limbs and stifle joint effusion if present (in case of concomitant CrCL lesion). Sometimes, locating the patella can be challenging; in these cases, the identification of the tibial tuberosity and then following the patellar ligament proximally can be helpful. Once located, the patella is isolated between the thumb and the index finger of one hand, the leg is extended and the other hand grabs the hock joint, or the foot, and twists the leg internally (medial luxation) or externally (lateral luxation). This test can be easily performed with the dog in lateral recumbence, because muscle tension can prevent luxating patella (Figure 2). In grade I and II luxation, the diagnostic test consists of pushing the patella out from the trochlear groove, differently in grades III and IV during the test the patella is attempted to be relocated in a normal position. ${ }^{20,22,27}$

During manipulation, it is possible to determine instability (in both directions), location of the patella, inability or ability to reduce the patella, presence of crepitus, range of motion, pain, and presence or absence of drawer movement. In dogs with severe skeletal deformities, tibial tuberosity deviation and limb torsion or angulation can be palpated. The depth of the trochlear groove can also be occasionally assessed by palpation as well the position of the patella within the trochlear groove. In some dogs, such as the Akita or Sharpei, the patella occasionally is riding too high in the trochlea ("patella alta"), whereas in chondrodystrophied dogs, it may be abnormally low ("patella baja"). ${ }^{20,27}$

If the dog does not cooperate, sedation may be required to perform an appropriate orthopedic examination. Although, under sedation, manipulation is facilitated by muscle relaxation, some grade IV luxations are characterized by such severe muscle contracture that full extension of the stifled joint is not achievable. ${ }^{20}$

\section{Diagnostic imaging}

Radiographic survey of the stifle helps to confirm luxation and assess degenerative changes present in the joint. It is also essential to identify any skeletal abnormality. Orthogonal views of the stifle may be sufficient when skeletal abnormalities are mild. However, if the luxation grade is high and severe skeletal deformity is present, additional orthogonal views of the femur and the tibia are required. ${ }^{22,29}$

Lateral projection allows assessing the correct positioning of the patella in the trochlear groove; in case of dislocation, the patella appears superimposed on the femoral condyles in a backward position. Craniocaudal projection allows identification of the patella medial or lateral to the distal aspect of the femur as well evaluating any varus or valgus deformity. Additional projections include axial view of the femur in order to assess the femoral torsion angle, or anteversion angle, and the skyline (tangential) view to evaluate the depth of the trochlear groove and the integrity of the trochlear ridges (Figure 3). ${ }^{22,29}$

Careful positioning is essential in order to obtain reliable radiological examinations. Sometimes, because of bone 


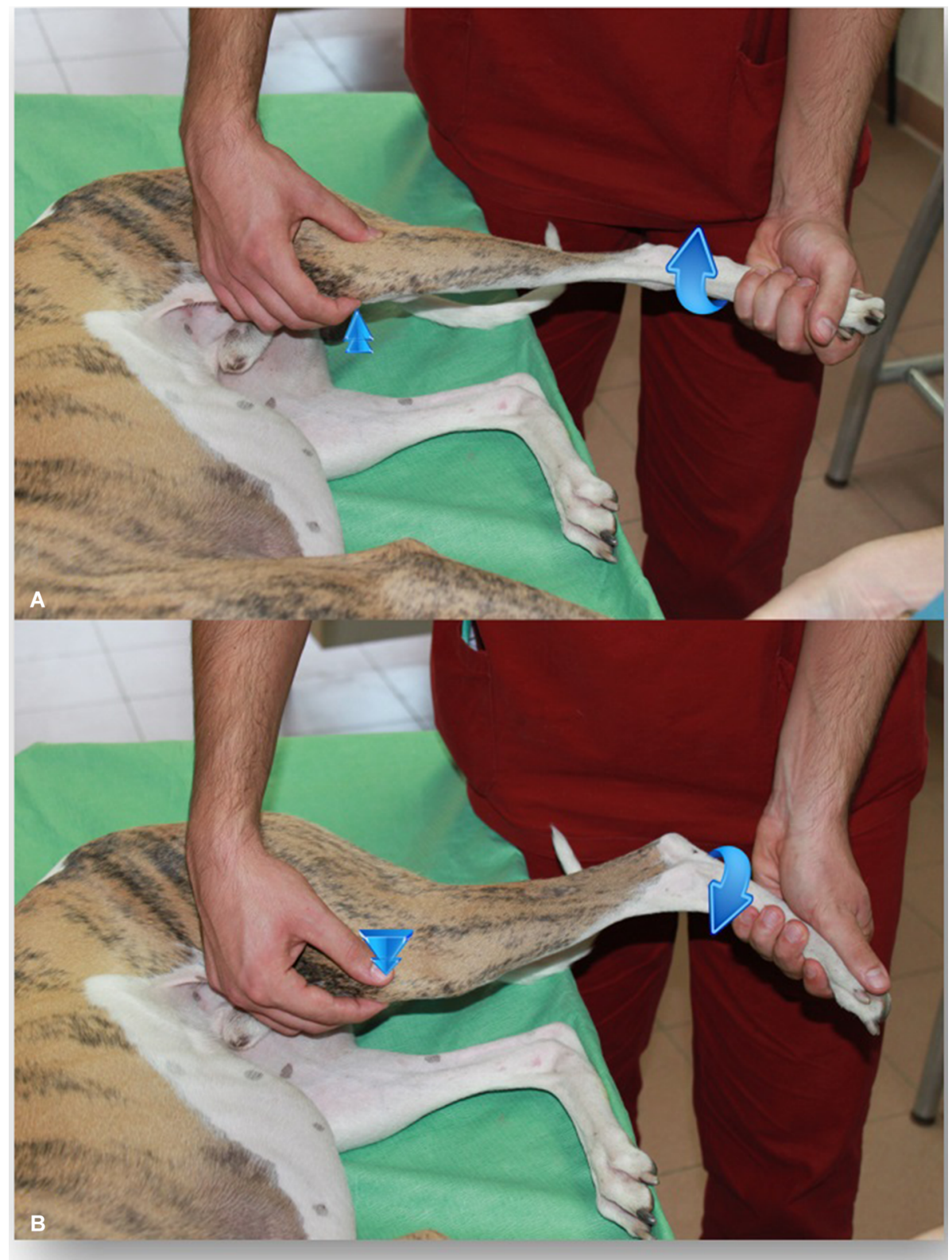

Figure 2 The pictures show the manipulation to luxate the patella.

Notes: The patella is isolated between the thumb and the index finger of one hand, while the leg is extended and the other hand grabs the foot. (A) Lateral luxation: the leg is twisted externally while the patella is pulled out laterally. (B) Medial luxation: the leg is twisted internally while the patella is pulled out medially.

deformities or muscle contracture, positioning of the dog could be difficult. Advanced diagnostic imaging, such as CT, is currently used to go beyond the limitations of conventional radiology (Figure 4). Reliable images and measurements can be obtained by using 3D reconstructions; also, the time required to perform this diagnostic procedure is much less compared with a radiographic survey. ${ }^{30-32}$ On both the radiographs and the CT scan, the anatomic and mechanical joint angles of the femur and tibia can be calculated to quantify the deformity and plan surgical correction. ${ }^{33-40}$
Recently, ultrasonography has been used to assess the depth of the trochlear groove. ${ }^{41}$

\section{Medical treatments for patellar luxation}

Conservative management may be indicated in dogs with grade I patellar luxation if the episode of lameness is mild and infrequent, and the degree of osteoarthritis is mild. Non-surgical management typically includes administration of nonsteroidal anti-inflammatory drugs (NSAIDs) in association, or not, with other analgesic drugs to reduce 

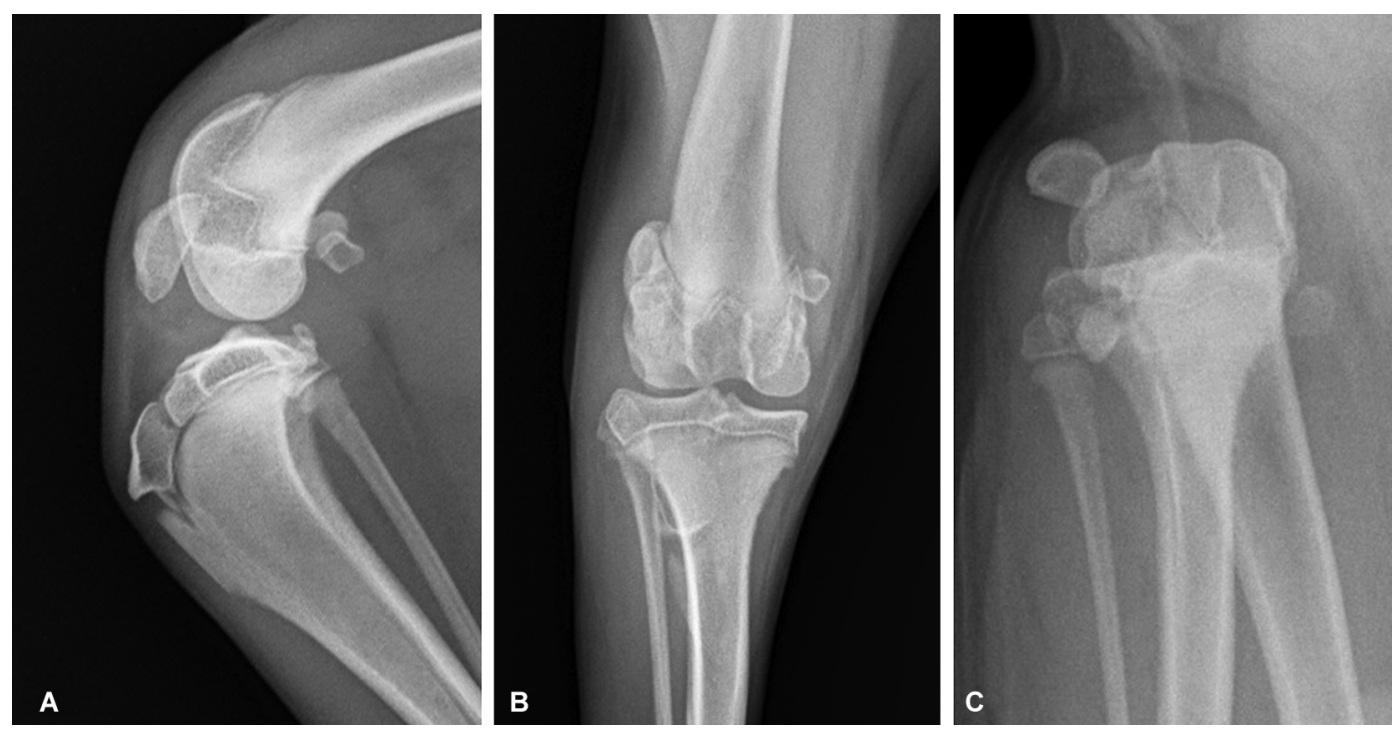

Figure 3 Mediolateral (A), craniocaudal (B), and tangential (C) radiographic projections of a canine stifle with lateral patellar luxation.
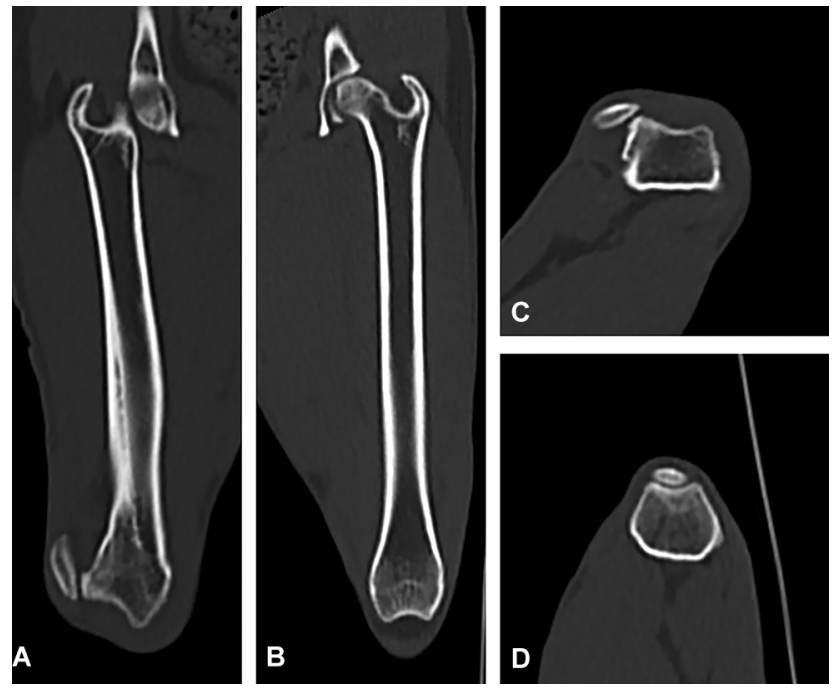

Figure 4 CT scan of a dog with unilateral lateral patellar luxation.

Notes: (A) Frontal view of the femur showing dislocation of the patella laterally and distal femoral bowing. (B) Normal. (C) Transverse view showing abnormal shape of the trochlear groove and lateral dislocation of the patella. (D) Normal.

pain. Physical rehabilitation exercises are useful to enhance quadriceps mechanism. Weight control is essential to reduce undue stress on the stifle joint. Also, massage therapy and hydrotherapy may help to promote wellness and comfort. ${ }^{27}$

\section{Surgical treatments for patellar luxation}

Surgical treatments are recommended for dogs with an intermittent or permanent lameness as a result of patellar luxation or in young dogs in an attempt to mitigate the negative effects of the condition on growing bone. Joint pathology increases with age and luxation grade, and surgical correction should be performed at the earliest opportunity to limit further development of skeletal abnormalities or DJD. ${ }^{5}$

The primary aim of surgery is to restore normal alignment of the quadriceps muscle relative to the entire limb. Surgical techniques can be divided into those that involve reshaping of the bones and those that involve only soft tissues reconstruction. Most of the dogs that undergo surgery need some combination of osseous and soft tissue techniques. $^{22}$

\section{Soft tissues techniques}

In most dogs affected by patellar luxation, the soft tissues on either side of the patella are either too tight or too loose. Reconstructions are usually performed to release tight tissues and tighten loose tissues. Soft tissue procedures include medial desmotomy, lateral imbrication, antirotational sutures, and release of medial musculature. These procedures are rarely able to correct patellar luxation, more often they are used to supplement osseous procedures., ${ }^{5,42}$

Desmotomy refers to release the soft tissues through an incision of the retinaculum on the medial or lateral side of the joint, according to the direction of the luxation. Sometimes, the releasing incision includes the joint capsule. ${ }^{20}$

Lateral imbrication involves the use of sutures to tighten up soft tissues contralateral to the luxation. This procedure is usually performed in association with desmotomy. ${ }^{5,9,22}$

Antirotational sutures are similar to the extracapsular sutures used for repair of the CrCL. A fabellotibial suture can be placed from the lateral fabella to the tibial crest, resulting in external rotation of the tibia and moving the 
tibial tuberosity into a more lateral position. Alternatively, a suture can be passed behind the fabella and then around the patella in order to create tension on the opposite side of the luxation. ${ }^{11,20,22}$

Muscle release can be achieved by dissecting the rectus femoris muscle away from the joint capsule, the femur and musculature, acting as an extension of the desmotomy. Freeing the rectus femoris muscle, at least to the level of the middle of the femur, may relieve significant medially directed tension on the patella. Another approach is to transplant the origins of the rectus femoris muscle or the cranial portion of the sartorius muscle to a more lateral locations reducing medial pull on the patella..$^{9,11,20}$

\section{Tibial tuberosity transposition}

Tibial tuberosity, or crest, transposition may be the most important component of surgical treatment for dogs with patellar luxation since quadriceps malalignment is a key feature in the development of all grades of patellar luxation. The aim of this repair is to realign the insertion of the tendon spanning between the patella and tibia. Because bones heal much more efficiently than tendons, the bone that this tendon is attached to is cut and moved to a more appropriate position. $^{20,22}$

The exposure of the tibial crest can be achieved through a lateral parapatellar approach to the stifle joint, by elevating the cranial tibial muscle. At this stage, the osteotomy can be performed using an osteotome and mallet or an oscillatory saw blade, leaving only the distal periosteal attachment. The crest is then transposed laterally or medially, according to the direction of the luxation, until the patella is centrally located within the trochlear groove. The crest can be also moved distally as needed to create patellar tendon tension that would aid in maintaining reduction. In some cases, the underlying tibia at the transplantation site needs to be prepared to expose cancellous bone and allow flat seating of the crest and this can be achieved by using an osteotome or a rasp. An appropriately sized Kirschner wire (0.035-0.062 inch) is then passed through the crest and into the tibia in a caudodistal direction until it is seated in the far cortex. A second Kirschner wire is usually used to increase the stability. A strand orthopedic wire (18-22 gauge), in a figure-of-eight pattern, is placed through a tunnel, caudal and slightly distal to the distal extent of the osteotomy, and on the Kirschner wires, creating a tension band (Figure 5). The Kirschner wires are bent over proximally and cut, about $3 \mathrm{~mm}$ beyond the bend, to leave a small palpable protrusion for later removal., 4,5,20,22,26,43

\section{Deepening of the trochlear groove}

Sulcoplasty techniques are used to modify the shape of the trochlear groove to accommodate the patella. When assessing whether to perform sulcoplasty or not, the surgeon has to consider that any sulcoplasty leads to unavoidable cartilage damage. For this reason, techniques that preserve the articular cartilage should be preferred. Many different approaches have been described to deepen the trochlear groove, including abrasion trochleoplasty, trochlear chondroplasty, and wedge and block recession trochleoplasty. The goal of all these techniques is to achieve a trochlear groove that is deep and wide enough to embed $\sim 50 \%$ of the patella above the trochlear ridges. ${ }^{20}$

Abrasion trochleoplasty is probably the simplest technique described and is characterized by the removal of the articular cartilage and several millimeters of subchondral bone by using a high-speed bur, a rongeur, or a bone rasp. The main disadvantage of this technique is the complete loss
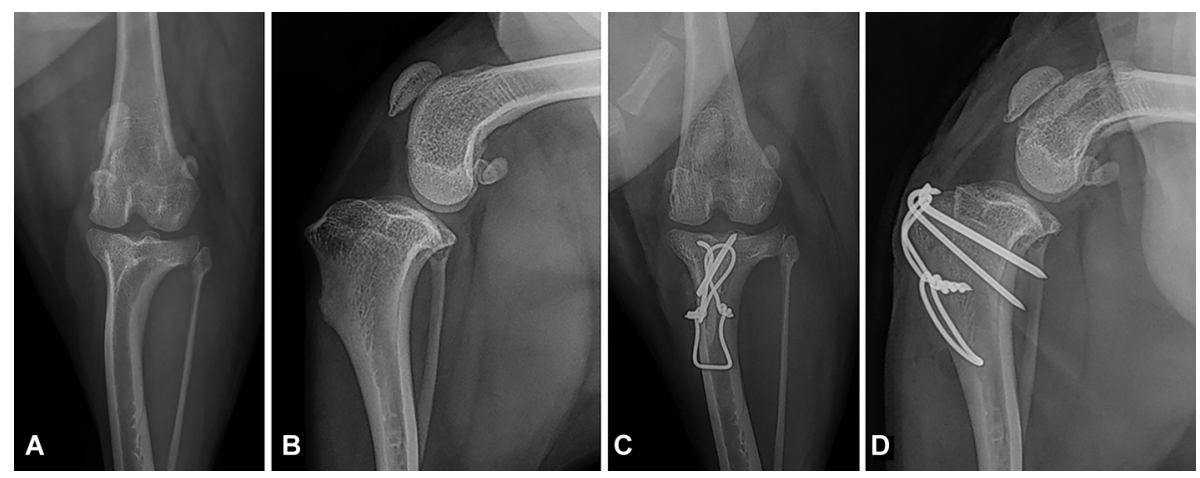

Figure 5 Craniocaudal (A) and mediolateral (B) radiographic projection of a canine stifle with medial patellar luxation. Craniocaudal (C) and mediolateral (D) radiographic projections of the same stifle after surgical correction.

Notes: It should be noted that pins and tension band wire were used to perform a tibial tuberosity transposition. A wedge recession sulcoplasty has been performed and mild radiographic changes can be observed on the postoperative mediolateral projection (D). The position of the patella appears to be straight with regard to the distal femur and the trochlear groove $(\mathbf{C})$ compared with the preoperative radiograph $(\mathbf{A})$. 
of the hyaline cartilage lining of the femoral trochlea, with subsequent erosion of the articular cartilage of the patella. ${ }^{44}$

Trochlear chondroplasty can be performed in dogs younger than 6 months by incising the articular cartilage of the trochlea with a scalpel blade at its proximal extent, and along the trochlear ridges. Then, using a periosteal elevator, the hyaline cartilage can be gently elevated from the underlying subchondral bone. At this stage, the trochlear groove is deepened by removing several millimeters of subchondral bone with a curette, rongeur, or rasp. ${ }^{5,20}$

The trochlear wedge and block recession techniques aim to achieve a deepening of the trochlear groove through the collection of an osteochondral autograft from the trochlear sulcus. This autograft can be triangular (wedge) or rectangular in cross-section. A fine-toothed saw is generally used to perform the osteotomies. Once the osteochondral autograft has been freed from the femur, the defect is deepened. When performing a wedge osteotomy, the subchondral bone must maintain the same apex angle during the deepening procedure because the original wedge fits exactly in the defect when replaced (Figure 6). When performing the block recession technique, the 2 osteotomies parallel to the trochlear ridges are connected through a straight basilar osteotomy using an osteotome. A slight angulation of each abaxial osteotomies toward the sagittal plane of the femur results in a press-fit of the rectangular osteochondral block and the recipient bed of the femoral trochlea. Both types of osteochondral autograft fits exactly into the larger defect, achieving immediate stability, recession of its articular surface, and a relative increase in height of the trochlear ridges. Adjunctive stability is due to retropatellar pressure and congruence between the cut surfaces; no internal fixation is needed. ${ }^{45-47}$

\section{Corrective osteotomies}

Corrective osteotomies of the distal femur and proximal tibia may be performed if there is significant femoral and/or tibial malalignment. Femoral varus is the most common abnormality in dogs with patellar luxation. Common procedures are lateral closing wedge ostectomy and medial opening wedge osteotomy. The first one consists of taking out a wedge of bone and then reducing and stabilizing the proximal and distal segments using plate and screws applied laterally. During this procedure, a standard tibial plateau leveling osteotomy jig is used to aid with reduction of the fragments. Two Kirschner wires can also be placed from distolateral to proximomedial and distomedial to proximolateral to maintain reduction. The ostectomized wedge of the distal femur can be morselized and used as an autogenous bone graft at the ostectomy site. The lateral closing wedge technique is a primary stable correction with a high rate of consolidation, but has the disadvantage of bone loss and can hesitate in limb shortening. 22,48

The medial opening wedge technique does not result in any bone loss but needs to be fixated with a medial plate (or double plating) and is characterized by a bone gap that can

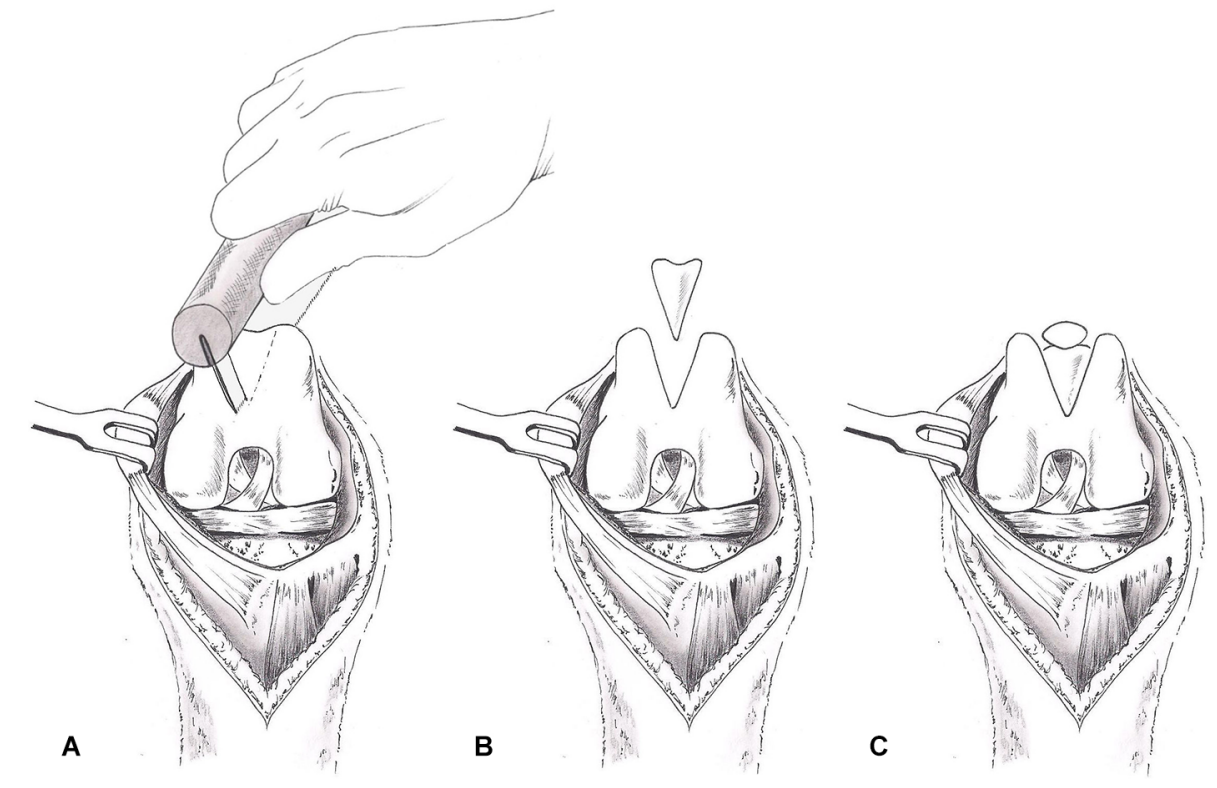

Figure 6 Schematic representation of wedge recession sulcoplasty.

Notes: (A) The trochlear groove is absent and a fine-toothed saw is used to perform the osteotomies; (B) once separated, the wedge is removed and several millimeters of the subchondral bone are removed to deepen the groove; $(\mathbf{C})$ the osteochondral graft is then replaced. It should be noted that at the end of the procedure, there is an adequately deep trochlear groove where the patella can seat properly. 
be filled with autologous cancellous bone graft. The opening wedge osteotomy has the advantage of avoiding leg shortening, but delayed union or nonunion may result. ${ }^{22}$

Detorsional osteotomies can be used to address torsional deformities of long bones. Metaphysis is the preferred site to perform an osteotomy due to the high rate of healing. Once the osteotomy is completed, the distal fragment of the femur (or the proximal fragment of the tibia) is rotated until achieving an appropriate limb alignment. The osteotomy is then fixed with plate and screws. ${ }^{21}$

Accurate preoperative measurement must be made to ensure the osteotomy location and magnitude of femoral/ tibial angulation and torsion. ${ }^{37} \mathrm{CT}$ scans are particularly important when planning such corrections and 3-dimensional reconstruction; also, manufactured bone models may help to achieve the best result. ${ }^{22,38}$

\section{Further treatments}

Hemiepiphysiodesis has been recently described for the treatment of proximal tibial valgus through reducing the growth of the medial proximal epiphysis. This technique should be considered as an early treatment for immature animals with medial patellar luxation due to proximal tibial deformity. Serial radiographs to monitor for correction are needed; also, implant removal should be considered if overcorrection occurs. ${ }^{49}$

An alternative to sulcoplasty when carrying out procedures used to correct patellar luxation is the RidgeStop ${ }^{\mathrm{TM}}$. It is a prosthetic ridge held in place using standard cortical screws to build up the sides of the patellar groove where it is insufficient or eroded due to degenerative conditions. ${ }^{50}$

Patellar groove replacement is a novel method of treating femoropatellar instability in association with severe femoropatellar osteoarthritis. This procedure is performed by substituting the femorotrochlear groove with a prosthesis. ${ }^{51}$

\section{Postoperative period}

A soft-padded bandage is generally placed for few days to reduce swelling, decrease pain, and prevent self-trauma to the incision. Postoperative orthogonal radiographs are useful to assess correction of the patellar luxation and adequate implant placement.

Activity should be restricted for 6-8 weeks. Exercise should initially be limited to short, slow lead walks; specific physical rehabilitation exercises, including range of motion, strength, postural, and flexibility exercises, can accelerate the recovery and prevent muscle mass deterioration. Radiographs should be obtained at 6-8 weeks to evaluate healing of the tibial crest transposition or corrective osteotomies. Once the osteotomies are healed, the animal could then be gradually returned to normal activity. Postoperative analgesia is generally provided by using NSAIDs; opioids are used as needed..$^{20,27}$

\section{Complications and prognosis}

Complications include patellar reluxation, delayed union, or fixation failure at the osteotomy sites, infection, implantassociated complications, and osteoarthritis. ${ }^{22}$

Implant-associated complications and reluxation are the most common complications, followed by tibial crest avulsion. ${ }^{52}$ Previous studies detected a correlation between body weight and risk of reluxation after corrective surgery. ${ }^{16,53}$ Discordant data have been reported about the association between single-session bilateral surgery and risk of complications. Some authors have identified single-session bilateral surgery, for lateral patellar luxation correction, as the only variable significantly associated with reluxation. ${ }^{54}$ Recent data demonstrated that in small breed dogs with bilateral medial patellar luxation, the complication rate of singlesession bilateral surgery was comparable with staged bilateral or unilateral medial patellar luxation surgery. ${ }^{55,56}$ Another study, evaluating the surgical management of medial patellar luxation in Pomeranian dogs, demonstrated that grade II luxation had good outcome with a $100 \%$ success rate following surgery; dogs with grade III luxation experienced recurrent patellar luxation in about $11 \%$ of the cases; while dogs with grade IV luxation had higher rate of reluxation, with $36 \%$ of the dogs subjected to surgery, because varying degree of skeletal deformities. ${ }^{23}$

\section{Conclusion}

Patellar luxation is extremely prevalent in small breed dogs and is one of the major cause of hindlimb lameness and early osteoarthritis development in the canine stifle. An early diagnosis is essential to prevent further development of the disease and severe secondary joint changes. However, the decision making could be challenging because the degree of skeletal deformity and osteoarthritis present, as well the potential for progression of osteoarthritis vary from case to case. According to the abnormality identified, individualized treatment is provided for each patient. The wide range of available techniques and its combination can provide adequate option for any kind of cause of patellar luxation.

The treatment for dogs with subclinical grade I and II medial patellar luxation could be conservative, but if the dog exhibits lameness, surgery should be considered. Differently, 
grade III and IV luxations need surgical correction to restore a normal quadriceps mechanism. The prognosis is favorable in most of the cases and the risk of complication, as well recurrence of the disease, is related to the grade of luxation and the severity of deformity.

\section{Acknowledgment}

The authors thank Fabio Esposito for his kind assistance with the figures.

\section{Disclosure}

The authors report no conflicts of interest in this work.

\section{References}

1. Nunamaker DM. Patellar luxation. In: Newton CD, Nunamaker DM, editors. Textbook of Small Animal Orthopaedics. Philadelphia: Lippincott; 1985:941-947.

2. DeAngelis M. Patellar luxation in dogs. Vet Clin North Am Small Anim Pract. 1996;1:403-415.

3. Ness MG, Abercromby RH, May C, Turner BM, Carmichael S. A survey of orthopaedic conditions in small animal veterinary practice in Britain. Vet Comp Orthop Traumatol. 1996;9(2):43-52.

4. Linney WR, Hammer DL, Shott S. Surgical treatment of medial patellar luxation without femoral trochlear groove deepening procedures in dogs: 91 cases (1998-2009). J Am Vet Med Assoc. 2011;238(9):1168-1172.

5. Roush JK. Canine patellar luxation. Vet Clin North Am Small Anim Pract. 1993;23(4):855-868.

6. Bosio F, Bufalari A, Peirone B, Petazzoni M, Vezzoni A. Prevalence, treatment and outcome of patellar luxation in dogs in Italy. A retrospective multicentric study (2009-2014). Vet Comp Orthop Traumatol. 2017;30(5):364-370.

7. Hayes AG, Boudrieau RJ, Hungerford LL. Frequency and distribution of medial and lateral patellar luxation in dogs: 124 cases (1982-1992). J Am Vet Med Assoc. 1994;205(5):716-720.

8. LaFond E, Breur GJ, Austin CC. Breed susceptibility for developmental orthopedic diseases in dogs. J Am Anim Hosp Assoc. 2002;38(5): 467-477.

9. L'Eplattenier HF, Montavon P. Patellar luxation in dogs and cats: management and prevention. Comp Cont Edu Pract Vet. 2002;24:292-300.

10. Di Dona F, Della Valle G, Balestriere C, et al. Lateral patellar luxation in nine small breed dogs. Open Vet J. 2016;6(3):255-258.

11. Harasen G. Patellar luxation: pathogenesis and surgical correction. Can Vet J. 2006;47(10):1037-1039.

12. Priester WA. Sex, size, and breed as risk factors in canine patellar luxation. J Am Vet Med Assoc. 1972;4(1):633-636.

13. Alam MR, Lee JI, Kang HS, et al. Frequency and distribution of patellar luxation in dogs. 134 cases (2000 to 2005). Vet Comp Orthop Traumatol. 2007;20(1):59-64

14. O'Neill DG, Meeson RL, Sheridan A, Church DB, Brodbelt DC. The epidemiology of patellar luxation in dogs attending primary-care veterinary practices in England. Canine Genet Epidemiol. 2016;3:4.

15. Vidoni B, Sommerfeld-Stur I, Eisenmenger E. Diagnostic and genetic aspects of patellar luxation in small and miniature breed dogs in Austria Comp Anim Pract. 2006;16:149.

16. Arthurs GI, Langley-Hobbs SJ. Complications associated with corrective surgery for patellar luxation in 109 dogs. Vet Surg. 2006;35(6): 559-566.

17. Bound N, Zakai D, Butterworth SJ, Pead M. The prevalence of canine patellar luxation in three centres. Clinical features and radiographic evidence of limb deviation. Vet Comp Orthop Traumatol. 2009;22(1):32-37.
18. Lavrijsen IC, Leegwater PA, Wangdee C, et al. Genome-wide survey indicates involvement of loci on canine chromosomes 7 and 31 in patellar luxation in flat-coated retrievers. BMC Genet. 2014;15(1):64

19. Soontornvipart K, Wangdee C, Kalpravidh M, et al. Incidence and genetic aspects of patellar luxation in Pomeranian dogs in Thailand. Vet J. 2013;196(1):122-125.

20. DeCamp CE, Johnson SA, Déjardin LM, Schaefer SL, editors. Brinker, Piermattei and Flo's Handbook of small Animal Orthopedics and Fracture Repair. 5th ed. St. Louis, MO, USA: Elsevier; 2015.

21. Petazzoni M. Corrective osteotomies in treating patellar luxation in large breed dogs. Proceedings of the European College of Veterinary Surgeons Annual Scientific Meeting; July 3-5, 2014; Copenhagen, Denmark. 175-176.

22. Kowaleski MP, Boudrieau RJ, Pozzi A. Stifle joint. In: Johnston SA, Tobias KM, editors. Veterinary Surgery Small Animal. 2nd ed. St. Louis, MO, USA: Elsevier Saunders; 2017:1071-1168.

23. Wangdee C, Theyse LF, Techakumphu M, Soontornvipart K, Hazewinkel HA. Evaluation of surgical treatment of medial patellar luxation in Pomeranian dogs. Vet Comp Orthop Traumatol. 2013;26(6):435-439.

24. Soparat C, Wangdee C, Chuthatep S, Kalpravidh M. Radiographic measurement for femoral varus in Pomeranian dogs with and without medial patellar luxation. Vet Comp Orthop Traumatol. 2012;25(3):197-201.

25. Fitzpatrick CL, Krotscheck U, Thompson MS, Todhunter RJ, Zhang Z. Evaluation of tibial torsion in Yorkshire Terriers with and without medial patellar luxation. Vet Surg. 2012;41(8):966-972.

26. Singleton WB. The diagnosis and treatment of some abnormal stifle conditions in the dog. Vet Rec. 1957;69(1):1387.

27. Pérez P, Lafuente P. Management of medial patellar luxation in dogs: what you need to know. Vet Ir J. 2014;4(12):634-640.

28. Campbell CA, Horstman CL, Mason DR, Evans RB. Severity of patellar luxation and frequency of concomitant cranial cruciate ligament rupture in dogs: 162 cases (2004-2007). J Am Vet Med Assoc. 2010;236(8): 887-891.

29. Marino DJ, Loughin CA. Diagnostic imaging of the canine stifle: a review. Vet Surg. 2010;39(3):284-295.

30. Apelt D, Kowaleski MP, Dyce J. Comparison of computed tomographic and standard radiographic determination of tibial torsion in the dog. Vet Surg. 2005;34(5):457-462.

31. Aper R, Kowaleski MP, Apelt D, Drost WT, Dyce J. Computed tomographic determination of tibial torsion in the dog. Vet Radiol Ultrasound. 2005;46(3):187-191.

32. Yasukawa S, Edamura K, Tanegashima K, et al. Evaluation of bone deformities of the femur, tibia, and patella in Toy Poodles with medial patellar luxation using computed tomography. Vet Comp Orthop Traumatol. 2016;29(1):29-38.

33. Banfield CM, Bartels JE, Hudson JA, Wright JC, Hathcock JT, Montgomery RD. A retrospective study of canine hip dysplasia in 16 military working dogs part 1: angle measurements and Orthopedic Foundation for Animals (OFA) grading. J Am Anim Hosp Assoc. 1996;32(5):413-422.

34. Hauptman J. Interobserver variation in the measurement of the femoral angle of inclination. Vet Surg. 1983;12(4):189-191.

35. Kaiser S, Cornely D, Golder W, Garner M, Waibl H, Brunnberg L. Magnetic resonance measurements of the deviation of the angle force generated by contraction of quadriceps muscle in dogs with congenital patellar luxation. Vet Surg. 2001;30(6):552-558.

36. Sarierler M. Comparison of femoral inclination angle measurements in dysplastic and nondysplastic dogs of different breeds. Acta Vet Hung. 2004;52(2):245-252.

37. Tomlinson J, Fox D, Cook JL, Keller GG. Measurement of femoral angles in four dog breeds. Vet Surg. 2007;36(6):593-598.

38. Dudley RM, Kowaleski MP, Drost WT, Dyce J. Radiographic and computed tomographic determination of femoral varus and torsion in the dog. Vet Radiol Ultrasound. 2006;47(6):546-552.

39. Dismukes DI, Tomlinson JL, Fox DB, Cook JL, Song KJ. Radiographic measurement of the proximal and distal mechanical joint angles in the canine tibia. Vet Surg. 2007;36(7):699-704. 
40. Dismukes DI, Tomlinson JL, Fox DB, Cook JL, Witsberger TH. Radiographic measurement of canine tibial angles in the sagittal plane. Vet Surg. 2008;37(3):300-305.

41. Hansen JS, Lindeblad K, Buelund L, Miles J. Predicting the need for trochleoplasty in canine patellar luxation using pre- and intraoperative assessments of trochlear depth. Vet Comp Orthop Traumatol. 2017;30(2):131-136.

42. Hulse DA. The stifle joint. In: Olmstead ML editor. Small Animal Orthopedics. St Louis, MO, USA: Mosby; 1995:395-404.

43. Robins GM. The canine stifle joint. In: Whittick WG, editor. Canine Orthopedics. 2nd ed. Philadelphia: Lea \& Febiger; 1990:693-760.

44. Vierheller RC. Surgical correction of patellar ectopia in the dog. J Am Vet Med Assoc. 1959;134(10):429-433.

45. Boone EG, Hohn RB, Weisbrode SE. Trochlear recession wedge technique for patellar luxation: an experimental study. J Am Anim Hosp Assoc. 1983;19:735.

46. Johnson AL, Probst CW, DeCamp CE, et al. Comparison of trochlear block recession and trochlear wedge recession for canine patellar luxation using a cadaver model. Vet Surg. 2001;30(2):140-150.

47. Slocum B, Slocum DB, Devine T, Boone E. Wedge recession for treatment of recurrent luxation of the patella. Clin Orthop Rel Res. 1982;164:48-53.

48. Brower BE, Kowaleski MP, Peruski AM, et al. Distal femoral lateral closing wedge osteotomy as a component of comprehensive treatment of medial patellar luxation and distal femoral varus in dogs. Vet Comp Orthop Traumatol. 2017;30(1):20-27.
49. Olsen AM, Vezzoni L, Ferretti A, Palmer RH, Vezzoni A, Duerr F. Hemiepiphysiodesis for the correction of proximal tibial valgus in growing dogs. Vet Comp Orthop Traumatol. 2016;29(4):330-337

50. RidgeStop ${ }^{\mathrm{TM}}$ patellar luxation repair system [homepage on the Internet]. Orthomed (UK) Ltd. Available from: https://www.orthomed.co.uk/. Accessed January 14, 2018.

51. Dokic Z, Lorinson D, Weigel JP, Vezzoni A. Patellar groove replacement in patellar luxation with severe femoro-patellar osteoarthritis. Vet Comp Orthop Traumatol. 2015;28(2):124-130.

52. Cashmore RG, Havlicek M, Perkins NR, et al. Major complications and risk factors associated with surgical correction of congenital medial patellar luxation in 124 dogs. Vet Comp Orthop Traumatol. 2014;27(4): 263-270.

53. Gibbons SE, Macias C, Tonzing MA, Pinchbeck GL, McKee WM. Patellar luxation in 70 large breed dogs. J Small Anim Pract. 2006;47(1):3-9.

54. Shaver SL, Mayhew KN, Sutton JS, et al. Complications after corrective surgery for lateral patellar luxation in dogs: 36 cases (2000-2011). J Am Vet Med Assoc. 2014;244(4):444-448.

55. Fullagar BA, Rajala-Schultz P, Hettlich BF. Comparison of complication rates of unilateral, staged bilateral, and single-session bilateral surgery for the treatment of bilateral medial patellar luxation in dogs. Can Vet J. 2017;58(1):39-44.

56. Gallegos J, Unis M, Roush JK, Agulian L. Postoperative complications and short-term outcome following single-session bilateral corrective surgery for medial patellar luxation in dogs weighing < $15 \mathrm{~kg}$ : 50 cases (2009-2014). Vet Surg. 2016;45(7):887-892.
Veterinary Medicine: Research and Reports

\section{Publish your work in this journal}

Veterinary Medicine: Research and Reports is an international, peer-reviewed, open access journal publishing original research, case reports, editorials, reviews and commentaries on all areas of veterinary medicine. The manuscript management system is completely online and includes a very quick and fair peer-review system.

\section{Dovepress}

Visit http://www.dovepress.com/testimonials.php to read real quotes from published authors. 